\title{
Thermal imaging and quantitative modelling of a laser diode for optical network communications
}

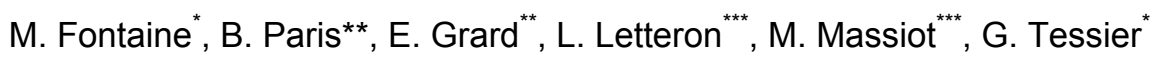 \\ *Institut Langevin, UMR CNRS 7587, ESPCI, 10 rue Vauquelin 75005 Paris, France, gilles.tessier@espci.fr \\ ** 3S Photonicsgroup, Route de Nozay, 91460 Marcoussis, France \\ ${ }^{* * *}$ Egide, P.A. de Pissaloup, 78190 Trappes, France
}

\begin{abstract}
Using infrared thermography, we have carried out a detailed characterization of the thermal properties of a laser module for optical telecommunications. In association with the measurement of thermal properties of its constitutive materials and thermoreflectance temperature imaging, this allowed us to validate a complete finite element-based model of the complete laser module, with its active Peltier cooling. The good correlation between simulation and measurements allows us to numerically explore the thermal transfer mechanisms of this active device, and predict optimized combinations of materials and architectures.
\end{abstract}

\section{Introduction}

First used for long distance connections that require very high information throughput, optical fibers gradually spread to lower telecommunication network levels, to increase the flow induced by the rise of Internet usages. Passive Optical Networks are short distance optical links deployed near the end user, for which high power laser emitters are necessary linked to high splitting ratio needed. Their reliability and power consumption (the diode is regulated in temperature, notably to maintain a suitable emission wavelength) are extremely dependent on their thermal behaviour. This implies the use of a Peltier cooler inside the laser module for the thermal stabilization of the diode.

Using infrared thermography, we have carried out a detailed characterization of the thermal properties of the Peltier elements, and then of the full moduleincorporating the laser diode. Coupled to high resolution temperature imaging by thermoreflectance, this allowed us to obtain a full description of the module, and validate a finite element model describing the electrical and thermal behaviour of the module.

\section{Electro-thermal characterization of the Peltier device}

The Peltier cooler device is responsible for a large portion of the total electrical consumption of the module. It turns out that the precision of the characteristics given by suppliers is not sufficient to achieve sufficient precision. Its main thermal contact with the environment is achieved through a metallic baseplate currently used in such packages (a FeNiCo alloy subsequently called Kovar $($ ) ). We therefore conducted a full thermal and electrical characterization of a naked Peltier with or without its baseplate, and separated from the rest of the laser module. This allowed us to describe the inbound $\left(Q_{C}\right)$ and outbound $\left(Q_{H}\right)$ thermal power flows in the Peltier :

$$
Q_{C}=\alpha I_{P} T_{C}-\frac{1}{2} R I_{P}^{2}-K\left(T_{H}-T_{C}\right) \quad Q_{H}=\alpha I_{P} T_{H}+\frac{1}{2} R I_{P}^{2}-K\left(T_{H}-T_{C}\right)
$$

With a the Seebeck coefficient in V.K $K^{-1}$, IP the Peltier current in $A, T_{C}$ the upper (cold) side temperature in $K, T_{H}^{\prime}$ the lower (hot) side temperature in $\mathrm{K}, \mathrm{R}$ the electrical resistance in $\Omega$, and $\mathrm{K}$ the thermal conductance in W. $\mathrm{K}^{-1}$.
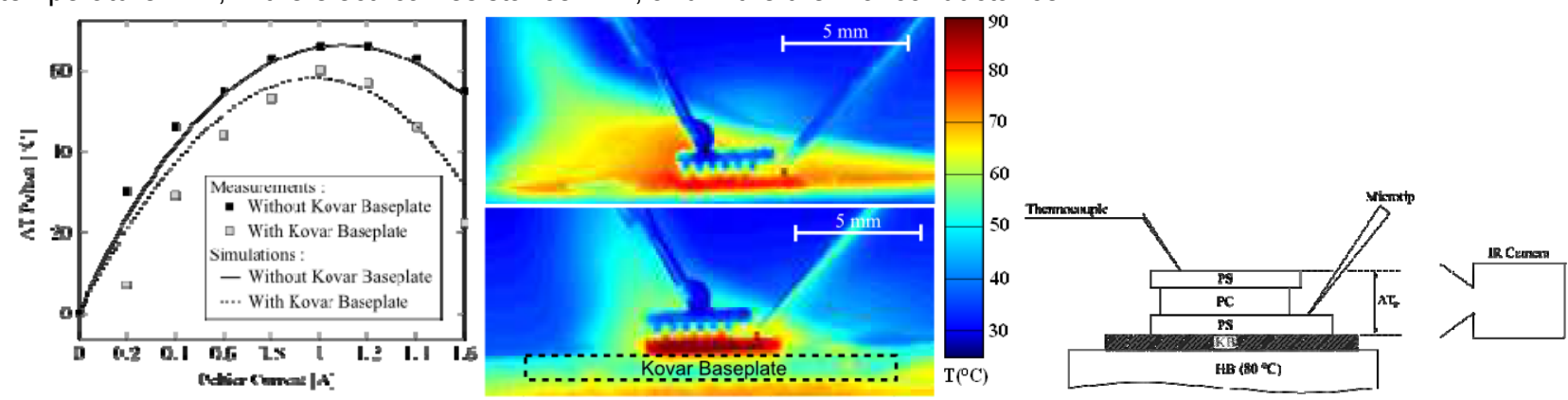

Fig. 1: (a) Measurements and simulations of a Peltier cooler with and without Kovar base plate. $\Delta T$ is the temperatures difference between the Hot base plate and the upper shelf of the Peltier cooler. (b) IR images of the Peltier module without and with Kovar base plate. (c) setup for the electro-thermal characterization of the Peltier cooler. 


\section{Thermal characterization of the complete laser module}

Heating induced by the laser is much easier to obtain, since it is simply the difference between the electrical consumption and the optical emission, which are both easily measured. To validate this and verify our hypothesis that all this power is dissipated in the laser ridge, high resolution thermoreflectance thermal imaging [1, 2].was carried out, allowing us to clearly identify the temperature distribution in the laser chip itself.

In order to obtain a uniform emissivity close to 1, the whole module was painted black. The infrared camera was then used to monitor temperature distribution on the surface of the complete moduleunder different operating conditions, as shown in Fig.2.

\section{Validation of the model}

A thermal characterization of the materials used in the metal package of the laser modules was also carried out using the Flash method, providing reliable values for the thermal conductivity and specific heat. These experimental values (electro-thermal properties of the Peltier and of the laser module, thermal properties of the metals) were then used to validate a finite element model of the full laser module based on the Comsol software package.

As shown in figure 2, the agreement between the temperature predictions and the IR measurements is good, and allowed us to confront the model to experimental conditions under various operating conditions (different base plate temperature, electrical power, laser operation temperature).

We will show that this validated model allows interesting predictive calculations to be carried out, allowing easy numerical prototyping and interesting improvements of the architecture to be obtained.

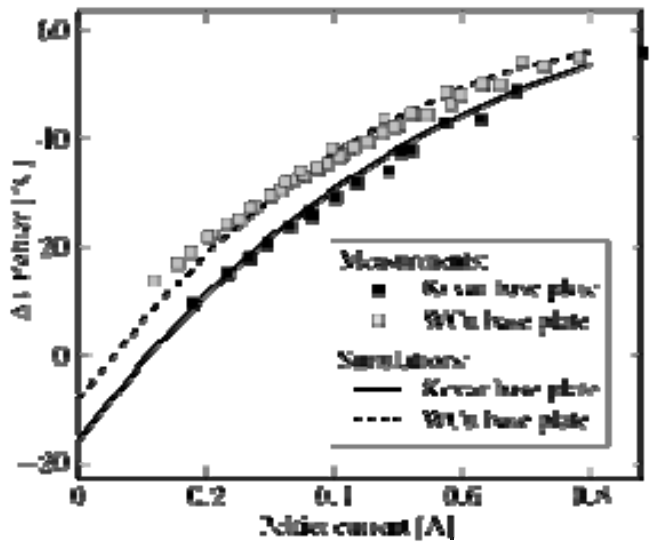

Fig. 2 : Measurements and simulations of temperatures difference of the complete system (laser, Peltier, casing) as a function of Peltier current, for two different base plate materials.

Acknowledgement : This work was partially funded by the Fonds Unique Interministeriel, FUI Trilob.

\section{REFERENCES}

[1] G. Tessier, S. Holé, D. Fournier, Quantitative thermal imaging by synchronous thermoreflectance with optimized illumination wavelengths, Applied Physics Letters 78, 2267 (2001).

[2] G. Tessier, G. Jerosolimski, S. Holé, D. Fournier, C. Filloy. Measuring and predicting the thermoreflectance sensitivity as a function of wavelength on encapsulated materials. Review of Scientific Instruments 74, 495 (2003) 